\title{
Thromboelastometry used for evaluation of blood coagulability in dogs with kidney diseases
}

\author{
Sara Falco ${ }^{1}$, Renato Zanatta ${ }^{1}$, Barbara Bruno ${ }^{1}$, Cristiana Maurella ${ }^{2}$, Aldo Scalone ${ }^{3}$, \\ Alberto Tarducci ${ }^{1}$, Antonio Borrelli ${ }^{1}$ \\ ${ }^{1}$ University of Torino, Department of Veterinary Science, Clinical Medicine Section, Grugliasco, Italy \\ ${ }^{2}$ Istituto Zooprofilattico Sperimentale del Piemonte, Liguria e Valle D’Aosta, Torino, Italy \\ ${ }^{3}$ Department of Infectious, Parasitic \& Immunomediated Diseases, Section of Vector-borne \\ Diseases \& International health, Istituto Superiore di Sanità, Rome, Italy \\ Received October 17, 2012 \\ Accepted April 23, 2013
}

\begin{abstract}
Kidney diseases as protein-losing nephropathies are predisposing conditions for thrombosis/ thromboembolism in dogs due to increased blood coagulability. The aim of this study was to use thromboelastometry for investigation of changes in haemostasis in dogs affected by renal proteinuria alone or concomitant with azotemia. The study was done at 55 client-owned, healthy $(n=34)$, proteinuric $(n=14)$, and proteinuric/azotemic $(n=7)$ dogs. Blood coagulation was assessed by means of standard coagulation profile and thromboelastometry (in-TEM, ex-TEM, and fib-TEM). In proteinuric dogs only the maximum clot firmness and maximum clot elasticity significantly increased $(P<0.05)$, whereas in the proteinuric/azotemic dogs the thromboelastometric profiles showed a pronounced increase of blood coagulability, as indicated by significant changes $(P<$ 0.05 ) of the variables clotting time, clot formation time, $\alpha$ angle, maximum clot firmness and maximum clot elasticity. After correction for group affiliation, the linear regression analysis showed a non significant association between changes in proteinuria and thromboelastometric profiles. In conclusion, thromboelastometric changes indicating hypercoagulabilty were more consistently found in the dogs with proteinuria/azotemia, than in those with proteinuria alone, suggesting that the former dogs can be considered at higher thrombotic risk. This is the first study where thromboelastometry has been applied to the investigation of haemostatic changes in dogs with kidney diseases.
\end{abstract}

Proteinuria, azotemia, viscoelastic technique, hypercoagulability

Kidney diseases are associated with the onset of altered haemostasis in dogs. Proteinlosing nephropathies (PLN) are a predisposing condition for thrombosis/thromboembolism. Among the three elements of Virchow's triad (blood flow stasis, hypercoagulability and endothelial injury), the last two are involved in the association between PLN and thromboembolism (Goggs et al. 2009). In proteinuric patients there is an imbalance between anticoagulant and procoagulant factors due to the urinary loss of antithrombin (AT) and other proteins, e.g., protein S and fibrinolytic proteins (Hull and Goldsmith 2008; Goggs et al. 2009). Furthermore, PLN can be associated with dyslipidaemia, inflammatory response, oxidative stress and endothelial dysfunction (Schiffrin et al. 2007); all of which can contribute to increased thrombotic risk.

The diagnosis of hypercoagulability is often difficult with routine coagulation profiles that can result normal even in dogs with evidence of pulmonary thromboembolism (Johnson et al. 1999). In contrast, the viscoelastic technique is a useful method to identify hypercoagulable patients (Donahue and Otto 2005). Viscoelastic techniques include sono-clot, thromboelastograph (TEG) and thromboelastometer (TEM). The devices measure the kinetics of clot formation, the tensile strength of the clot, and the rate of fibrinolysis. Coagulation is assessed globally, from the first phase of clot formation to the final lysis of the clot (McMichael and Smith 2011). Furthermore, the viscoelastic technique is a whole blood assessment that considers the role of plasmatic haemostatic

Address for correspondence:

Sara Falco, DMV, $\mathrm{PhD}$

Department of Veterinary Science

via Leonardo da Vinci 44, 10095 Grugliasco (TO), Italy
Phone: +390116709080

E-mail: sara.falcovet@gmail.com

http://actavet.vfu.cz/ 
components as well as platelets and cellular surfaces, and better reflects the cell-based model of coagulation (Smith 2009). A recent study has identified hypercoagulability by means of non-activated TEG in 7 dogs affected by renal failure and in 11 dogs with severe proteinuria (Donahue et al. 2011). Thromboelastometry on canine whole blood has also been recently validated (Smith et al. 2010).

The aim of this study was to analyze haemostatic changes in dogs affected by renal proteinuria and/or renal failure by means of both TEM and standard coagulation assay. The correlation between the magnitude of proteinuria and azotemia and the degree of haemostatic impairment was evaluated in order to identify those canine patients with higher hypercoagulability and at a potentially higher risk for thrombosis.

\section{Materials and Methods}

Study material

The study protocol was approved by the local Bioethics and Animal Welfare Committee (protocol number 1061/2012). The owners of all dogs recruited for participation in the study gave their informed consent after being informed about the risks of the procedures. A total of 55 dogs used in study were subdivided into three groups: control group of healthy dogs (group $\mathrm{C}, \mathrm{n}=34$ ), proteinuria group (group $\mathrm{P}, \mathrm{n}=14$ ) and proteinuria/ azotaemia group (group $\mathrm{F}, \mathrm{n}=7$ ). Group $\mathrm{C}$ was composed of 19 males and 15 females, group $\mathrm{P}$ of 10 males and 4 females and group F of 3 males and 4 females. The median age of dogs was: 4.5 years (range 1-15) in group C, 9 years (range 5-15) in group $P$ and 10 years (range 7-13) in group F. The most represented breeds were mixed breed (29, 9 and 3 in group $\mathrm{C}, \mathrm{P}$ and F, respectively) and Boxer (1, 2, 1 in group C, P and F). All dogs included in group $\mathrm{C}$ were deemed healthy on the basis of complete clinical history, physical examination, haematology and biochemistry. Dogs were included in group P if creatinine was $<125 \mu \mathrm{mol} / 1$ (i.e., stage 1 of the International Renal Interest Society (IRIS) staging system, Elliot and Watson 2008) and if persistent proteinuria of renal origin and a urine protein-to-creatinine (UPC) ratio $\geq 0.5$ were identified. Dogs were included in group F if they presented isostenuria, a UPC ratio $\geq 0.5$, and creatinine $\geq 125 \mu \mathrm{mol} / 1$ (i.e., IRIS stage from 2 to 4 , E1liot and Wats on 2008). Exclusion criteria were: body weight $<5 \mathrm{~kg}$, administration of anticoagulant or anti-inflammatory drugs (both NSAIDs and corticosteroids), postrenal proteinuria, neoplasia, hepatic disease or Cushing syndrome at the time of inclusion, positive tests for vector-borne diseases.

\section{Chemical examination}

A volume of $13 \mathrm{ml}$ whole blood was collected from each dog by jugular venipuncture using minimum stasis and minimizing blood vessel injury. The following laboratory tests were performed: complete blood count (CBC) (ADVIA 120 Hematology, System Siemens Diagnostics, Milan, Italy), blood biochemistry (ILAB 300 plus, Clinical Chemistry System, Instrumentation Laboratories, Milan, Italy), serum protein electrophoresis, IFAT serology (in-house method) for Leishmania infantum and immunochromatographic tests (Snap 4 DX, IDEXX Laboratories, Westbrook, Maine) for other vector-borne diseases such as: Ehrlichia canis, Borrelia burgdorferi, Dirofilaria immitis and Anaplasma phagocytophilum, coagulation profile (Coagulometer StART, Diagnostica Stago, Roche, Monza, Italy), including prothrombin time (PT), activated partial thromboplastin time (aPTT), fibrinogenaemia, AT and D-dimer (NycoCard D-dimer test, AXIS-SHIELD PoC AS, Oslo, Norway) and TEM (ROTEM, TEM innovation GmbH, Munich, Germany).

Urine samples were collected by cystocentesis. A urine dip-stick test was performed, the urine specific gravity was measured with a hand refractometer, the urine sediment was examined microscopically, the urine protein concentration was measured with pyrogallol red, the creatinine with the Jaffé method, and the UPC ratio was calculated.

Fine needle aspiration of popliteal lymph nodes was performed for the direct diagnosis of Leishmania infantum: cytologic evaluation or, when available, polymerase chain reaction (PCR) assay was performed.

Thromboelastometry was evaluated on citrated blood samples within $30 \mathrm{~min}$ after blood collection according to the manufacturer's instructions, and the analyses were run for $60 \mathrm{~min}$. In-TEM, ex-TEM and fib-TEM profiles for each sample were performed to evaluate the intrinsic pathway (with activation by ellagic acid), the extrinsic pathway (with tissue factor activation) and fibrinogen function (platelets inactivated with cytocalasine D), respectively. The following variables were assessed for each profile: clotting time ([CT], s); clot formation time ([CFT], s); maximum clot firmness $([\mathrm{MCF}], \mathrm{mm}) ; \alpha$ angle $\left(\alpha,{ }^{\circ}\right)$; and maximum clot elasticity $(\mathrm{MCE}) . \mathrm{MCE}$ describes the mechanical properties of the clot and is calculated from the MCF as follows: $\left(\mathrm{MCF}^{*} 100\right) /(100-$ MCF) (Lang et al. 2009). Finally, the platelet component of clot strength was evaluated by means of the variable $\mathrm{MCE}_{\text {platelet}}$, obtained as follows: $\mathrm{MCE}_{\text {platelet }}=\mathrm{MCE}_{\text {extem }}-\mathrm{MCE}_{\text {fibtem }}$ (Lang et al. 2009).

The data were checked for normality by the Shapiro-Wilk test. Whether the assumption of normality was not fulfilled, non parametrical test (Kruskal-Wallis rank test) was performed to investigate the distribution of the haematological, biochemical and haemostatic variables for the three groups; otherwise, one way analysis of variance with Bonferroni correction was computed. For any significant difference identified by KruskalWallis test, a pairwise comparison procedure was performed using Wilcoxon rank sum test. Linear regression 
analysis was used to determine whether any single variable correlated with the magnitude of proteinuria after having logarithmically transformed the UPC ratio. The association was adjusted for group of affiliation. To verify whether the platelet count was correlated with the variable $\mathrm{MCE}_{\text {platelet }}$, Pearson's linear correlation coefficient was calculated.

Significance was set at $P<0.05$. Statistical analysis was performed using the Stata software package ver. 11.2 MP.

\section{Results}

No significant difference in packed cell volume (PCV) or platelet count among the groups of dogs was found. The median creatinine level was $87.1 \mu \mathrm{mol} / 1$ (range from 55.4 to $118.8 \mu \mathrm{mol} / \mathrm{l}$ ) in group $\mathrm{C}, 83.6 \mu \mathrm{mol} / \mathrm{l}$ (range from 66.9 to $119.7 \mu \mathrm{mol} / \mathrm{l}$ ) in group $\mathrm{P}$, and $221.8 \mu \mathrm{mol} / \mathrm{l}$ (range from 127.6 to $903.8 \mu \mathrm{mol} / \mathrm{l}$ ) in group F. Two dogs in group F were classified as IRIS 2, 2 as IRIS 3 and 3 as IRIS 4. Albumin was significantly lower in group $\mathrm{P}$ compared to group $\mathrm{C}(P=0.008)$, whereas no significant difference emerged between group $\mathrm{F}$ and the other groups. Cholesterol was significantly higher in group $\mathrm{P}$ than in group $\mathrm{C}(P<0.001)$ and significantly higher in group $\mathrm{F}$ than in group $\mathrm{C}(P<0.001)$.

The UPC ratio ranged from 0.015 to 0.19 in group $C$, from 0.55 to 8.5 in group $\mathrm{P}$, and from 0.7 to 4 in group $\mathrm{F}$.

The results of the comparison of the haemostatic variables among the three groups are shown in Table 1. The crude linear regression analysis showed that most of the haemostatic variables resulted significantly associated with variations in the UPC ratio. After adjustment by group of affiliation, however, most of the associations lost their significance. The only variables still associated with UPC ratio variation were fibrinogen $(P=0.045)$ and AT $(P=0.042)$. None of the thromboelastometric variables showed a significant association with UPC ratio variations; however, in in-TEM MCF, in-TEM MCE, ex-TEM MCF and fib-TEM MCF the association was noted in group F $(P=0.023, P=0.016, P=0.026$, $P=0.04$, respectively).

A moderately significant correlation was found between platelet count and $\mathrm{MCE}_{\text {platelet }}$ $(P<0.05)$.

\section{Discussion}

The present study identified by means of TEM a hypercoagulable state in dogs affected by azotaemia and proteinuria and moderate haemostatic changes in proteinuric dogs.

In thromboelastometry, blood hypercoagulability is indicated by shortening of CT and CFT and increases in $\alpha, \mathrm{MCF}$ and MCE. Shortening of CT is suggestive of more rapid fibrin formation. This indicator is profoundly impacted by the activator used, whereas the other variables are less or minimally influenced (Smith et al. 2010). With the use of inTEM or ex-TEM reagents, the two coagulation pathways can be investigated separately because initial thrombin formation occurs exclusively via the intrinsic or the extrinsic pathway, respectively. Furthermore, the use of strong activators decreases the impact of sample changes occurring prior to initiation of the analysis, so that the results are more easily repeatable (Smith et al. 2010).

The phase of clot initiation in group P did not seem to be affected, as supported by the lack of significant alterations of CT, PT and aPTT in this group as compared to the healthy dogs. This last result contrasts with the results of a previous study conducted with non activated TEG (Donahue et al. 2011). A possible explanation for this difference is that we used strong contact activators that yield more repeatable results but also limit the procedure's sensitivity to subtle changes. Shortening of CT was observed in group F, but only on the ex-TEM profile, whereas the initial phase of the intrinsic pathway slowed down, as indicated by the prolongation of aPTT and the in-TEM CT. Further studies on a larger population with quantitative assessment of specific clotting factors and coagulation 
Table 1. Results of the comparison of haemostatic indicators among the study groups of dogs.

\begin{tabular}{|c|c|c|c|}
\hline \multirow[t]{2}{*}{ Haemostatic indicators } & \multicolumn{3}{|c|}{ Group of dogs } \\
\hline & C (control) & $\mathrm{P}$ (proteinuria) & F (proteinuria/azotaemia) \\
\hline PT & 6.9 & 7.2 & 6.8 \\
\hline$(\mathrm{sec})$ & $(5.8-9.3)$ & $(6.1-11)$ & $(6.3-8.7)$ \\
\hline aPTT & 11.5 & 11.4 & 12.7 \\
\hline$(\mathrm{sec})$ & $(10.1-15.3)$ & $(6.9-14.1)$ & $(12.1-16.1)^{\mathrm{ab}}$ \\
\hline Fibrinogen & 6.9 & $9.3^{\mathrm{a}}$ & 15.2 \\
\hline$(\mu \mathrm{mol} / 1)$ & $(3.9-13.7)$ & $(6.3-31.3)$ & $(9.3-27.8)^{\mathrm{b}}$ \\
\hline \multirow{2}{*}{ Antithrombin } & 127 & 114.5 & 94 \\
\hline & $(106-166)$ & $(72.1-148)$ & $(73-127)$ \\
\hline$(\%)$ & $129 \pm 14.8$ & $111.4 \pm 23.9^{\mathrm{a}}$ & $97.9 \pm 19^{\mathrm{a}}$ \\
\hline D-dimers & 100 & 200 & 150 \\
\hline$(\mathrm{ng} / \mathrm{ml})$ & $(50-500)$ & $(50-500)$ & $(50-200)$ \\
\hline In-TEM & $\mathrm{C}$ (control) & $\mathrm{P}$ (proteinuria) & $\mathrm{F}$ (proteinuria/azotaemia) \\
\hline \multirow{2}{*}{$\mathrm{CT}(\mathrm{sec})$} & 171 & 181 & 207 \\
\hline & $(126-346)$ & $(149-409)$ & $(167-608)^{\mathrm{a}}$ \\
\hline \multirow{2}{*}{ CFT (sec) } & 96 & 78 & 69 \\
\hline & $(49-189)$ & $(44-196)$ & $(32-201)^{\mathrm{a}}$ \\
\hline \multirow{2}{*}{$\alpha$ angle $\left({ }^{\circ}\right)$} & 72 & 75.5 & 77 \\
\hline & $(56-81)$ & $(54-81)$ & $(54-83)^{\mathrm{a}}$ \\
\hline \multirow{3}{*}{$\mathrm{MCF}(\mathrm{mm})$} & 62 & 65 & 74 \\
\hline & $(51-75)$ & $(57-78)$ & $(64-83)$ \\
\hline & $61.2 \pm 5.5$ & $65.4 \pm 6.7$ & $72.7 \pm 6.2^{\mathrm{a}}$ \\
\hline \multirow{2}{*}{$\mathrm{MCE}$} & 163.2 & 186 & 284.6 \\
\hline & $(104.1-300)$ & $(132.6-354.6)$ & $(177.8-488.2)^{\mathrm{ab}}$ \\
\hline Ex-TEM & $\mathrm{C}$ (control) & $\mathrm{P}$ (proteinuria) & F (proteinuria/azotaemia) \\
\hline \multirow{2}{*}{$\mathrm{CT}(\mathrm{sec})$} & 50 & 49.5 & 36 \\
\hline & $(34-92)$ & $(36-62)$ & $(27-48)^{\mathrm{ab}}$ \\
\hline \multirow{3}{*}{ CFT (sec) } & 115 & 88.5 & 56 \\
\hline & $(54-142)$ & $(33-170)$ & (32-93) \\
\hline & $109 \pm 24.3$ & $93.6 \pm 33.3$ & $55.4 \pm 20.3^{\mathrm{ab}}$ \\
\hline \multirow{3}{*}{$\alpha$ angle $\left({ }^{\circ}\right)$} & 68 & 73 & 81 \\
\hline & $(63-79)$ & $(58-83)$ & $(72-84)$ \\
\hline & $69 \pm 4.7$ & $71.9 \pm 6.2$ & $80 \pm 4^{\mathrm{ab}}$ \\
\hline \multirow{3}{*}{$\mathrm{MCF}(\mathrm{mm})$} & 60 & 65 & 73 \\
\hline & $(46-73)$ & $(54-82)$ & $(67-81)$ \\
\hline & $60.4 \pm 6.3$ & $65.6 \pm 7.1$ & $73 \pm 4.6^{\mathrm{a}}$ \\
\hline \multirow{2}{*}{ MCE } & 150.2 & 185.7 & 270.4 \\
\hline & $(85.2-270.4)$ & $(117.4-455.6)^{\mathrm{a}}$ & $(203-426.3)^{\mathrm{ab}}$ \\
\hline Fib-TEM & C (control) & $\mathrm{P}$ (proteinuria) & $\mathrm{F}$ (proteinuria/azotaemia) \\
\hline \multirow{2}{*}{$\mathrm{CT}(\mathrm{sec})$} & 43.5 & 47 & 34 \\
\hline & $(14-102)$ & $(17-60)$ & $(30-48)$ \\
\hline \multirow{3}{*}{$\alpha$ angle $\left({ }^{\circ}\right)$} & 62 & 70 & 83 \\
\hline & $(48-78)$ & $(55-82)$ & $(75-85)$ \\
\hline & $63.3 \pm 8.5$ & $69.1 \pm 8.1$ & $81 \pm 3.9^{\mathrm{ab}}$ \\
\hline
\end{tabular}


Table 1. Results of the comparison of haemostatic indicators among the study groups of dogs.

\begin{tabular}{lccc}
\hline Haemostatic indicators & & Group of dogs & \\
Fib-TEM & $\mathrm{C}$ (control) & $\mathrm{P}$ (proteinuria) & $\mathrm{F}$ (proteinuria/azotaemia) \\
\hline MCF $(\mathrm{mm})$ & 12 & 16.5 & 27 \\
& $(8-26)$ & $(10-38)^{\mathrm{a}}$ & $(16-37)^{\mathrm{ab}}$ \\
$\mathrm{MCE}$ & 13.6 & 19.8 & 37 \\
& $(8.7-35.1)$ & $(11.1-61.3)^{\mathrm{a}}$ & $(19.1-58.7)^{\mathrm{ab}}$ \\
MCE $_{\text {platelet }}$ & 135.1 & 167.8 & 243.8 \\
& $(75.3-240.5)$ & $(98.3-394.3)^{\mathrm{a}}$ & $(184-367.6)^{\mathrm{ab}}$ \\
\hline
\end{tabular}

Groups of dogs: $\mathrm{P}$ - dogs with proteinuria, F - dogs with proteinuria/azotaemia. Values are expressed as median (minimum-maximum) and, for values normally distributed, also as mean \pm standard deviation; ${ }^{\text {a }}$ indicates a significant difference $(P<0.05)$ with group $\mathrm{C}$; ${ }^{\mathrm{b}}$ indicates a significant difference $(P<0.05)$ with group $\mathrm{P}$. PT - prothrombin time, aPTT - activated partial thromboplastin time, CT - clotting time, CFT - clot formation time, MCF - maximum clot firmness, MCE - maximum clot elasticity

inhibitors are needed to better clarify this result and to investigate its clinical significance and possible correlation with uremic bleeding.

Our results also indicate an increase in the rapidity of clot development and amplification (i.e., shorter CFT and increased $\alpha$ angle) in group F, whereas this alteration was not observed in group P. This result contrasts with that reported by Donahue et al. (2011) and is probably due to differences in inclusion criteria: while Donahue et al.'s study included only dogs with a UPC $>2$, ours included especially dogs with moderate proteinuria $(0.5 \leq \mathrm{UPC}<2)$ that have shown moderate haemostatic changes. The following phase of clot stabilization, as represented by MCF and MCE, was the only haemostatic change that was significantly different in both groups $\mathrm{F}$ and $\mathrm{P}$. With the use of fib-TEM activator, which inhibits platelets, the contribution of functional fibrinogen to altering this phase in nephropathic dogs has been confirmed because significant alterations were found not only on the ex-TEM but also on the fib-TEM profile. The clot strength as measured by TEG or TEM increases in humans (Lang et al. 2009) and in dogs (Wiinberg et al. 2008) in a fibrinogen concentration-dependent manner. For this reason, fibrinogenaemia could be considered a predictor of increased clot stability.

Although TEM is not the most accurate device to investigate platelet function, it provides data supporting the hypothesis for a potential contribution of platelet function to hypercoagulability in nephropathic dogs, as indicated by the increased $\mathrm{MCE}_{\text {platelet }}$ in groups $\mathrm{P}$ and $\mathrm{F}$. This variable in human patients is not linearly related to platelet count because it increases with increasing platelet count and reaches a plateau at platelet count $>400 * 10^{3} / \mathrm{mm}^{3}$ (Lang et al. 2009). This correlation has not been investigated in canine patients so far. We found a moderate correlation between $\mathrm{MCE}_{\text {platelet }}$ and platelet count, with the latter not significantly different across the groups: this suggests an increase in platelet function in proteinuric dogs and a further increase in dogs with concomitant proteinuria and azotaemia. Specific studies investigating platelet function are needed to confirm this result.

The thromboelastometric results are affected also by the PCV. Specifically, low PCV leads to tracings indicating hypercoagulable states, whereas high PCV indicates hypocoagulability (McMichael and Smith 2011). This effect can pose a potential limitation when TEM is performed in dogs with diseases as renal failure which can also lead to anaemia. In the present study, however, we found no significant differences in PCV among the study groups. 
The results of linear regression emphasize the importance of azotaemia in haemostasis changes in our population. The lack of a significant correlation between proteinuria and degree of hypercoagulability could, again, be explained by the generally low degree of proteinuria in the group $\mathrm{P}$ dogs. Further studies are needed to determine whether there is a positive correlation with severe glomerular proteinuria.

A limitation of this study is the small population and the lack of a category of azotaemicnot-proteinuric dogs. Further studies on a larger population are needed to elucidate the relationship between kidney diseases and coagulation.

In conclusion, our study identified by means of TEM a hypercoagulable state in proteinuric and azotaemic dogs and moderate changes in proteinuric dogs. In the first group, the haemostatic changes involved all the thromboelastometric profiles and all the phases of clot formation and stabilization. For these reasons, dogs with proteinuria and concomitant azotaemia can be considered at higher risk for thrombotic/thromboembolic events. Further clinical studies are needed to better characterize the clinical relevance of TEM alterations and its correlation with thromboembolic risk and to inform the design of specific therapeutic protocols.

\section{Acknowledgements}

This study was not supported by any funding or grants.

\section{References}

Donahue SM, Brooks M, Otto CM 2011: Examination of hemostatic parameters to detect hypercoagulability in dogs with severe protein-losing nephropathy. J Vet Emerg Crit Care 21: 346-355

Donahue SM, Otto CM 2005: Thromboelastography: a tool for measuring hypercoagulability, hypocoagulability, and fibrinolysis. J Vet Emerg Crit Care 15: 9-16

Elliot J, Watson ADJ 2008: Chronic kidney disease: staging and management. In: Bonagura JD, Twedt DC (Eds.): Kirk's current veterinary therapy XIV. Saunders, St Louis, pp. 883-892

Goggs R, Benigni L, Fuentes VL, Chan DL 2009: Pulmonary thromboembolism. J Vet Emerg Crit Care 19: $30-52$

Hull RP, Goldsmith DJA 2008: Nephrotic syndrome in adults. BMJ 336: 1185-1189

Johnson LR, Lappin MR, Baker DC 1999: Pulmonary thromboembolism in 29 dogs: 1985-1995. J Vet Intern Med 13: 338-345

Lang T, Johanning K, Metzler H, Piepenbrock S, Solomon C, Rahe-Meyer N, Tanaka KA 2009: The effects of fibrinogen levels on thromboelastometric variables in the presence of thrombocytopenia. Anesth Analg 108: $751-758$

McMichael MA, Smith SA 2011: Viscoelastic coagulation testing: technology, applications, and limitations. Vet Clin Pathol 40: 140-153

Schiffrin EL, Lipman ML, Mann JFE 2007: Chronic kidney disease: effects on the cardiovascular system. Circulation 116: 85-97

Smith SA 2009: The cell-based model of coagulation. J Vet Emerg Crit Care 19: 3-10

Smith SA, McMichael M, Galligan A, Gilor S, Hoh CM 2010: Clot formation in canine whole blood as measured by rotational thromboelastometry is influenced by sample handling and coagulation activator. Blood Coagul Fibrinolysis 21: 692-702

Wiinberg B, Jessen LR, Johansson PI, Rozanski E, Tranholm M, Kristensen AT 2008: Thromboelastographic evaluation of hemostatic function in dogs with disseminated intravascular coagulation. J Vet Intern Med 22: $357-365$ 